\title{
Detection of Micro- to Nano-Sized Particles in Soft Tissue
}

\author{
Helmut Troster ${ }^{3}$, Stefan Milz ${ }^{2}$, Michael F. Trendelenburg ${ }^{3}$, F. Jorder ${ }^{1}$, \\ Hanns-Peter Scharf ${ }^{1}$, and Markus Schwarz ${ }^{1}$
}

\footnotetext{
${ }^{1}$ Lab. of Biomechanics and Exptl. Orthopaedics, Dep. of Orthopaedic Surgery, Faculty of Clinical Medicine Mannheim, University Heidelberg, Theodor-Kutzer-Ufer 1-3, D-68167

Mannheim, markus.schwarz@ortho.ma.uni-heidelberg.de

${ }^{2}$ University of Munich, Anatomical Institute, Pettenkofer Strasse 11, D-80336 München

${ }^{3}$ German Cancer Research Center (DKFZ), Biomedical Structure Analysis, Dep. A120, Im Neuenheimer Feld 280, D-69120 Heidelberg
}

\begin{abstract}
The demand is growing to study particulate debris in the human organism. This task is time-consuming. Therefore it is important to find an efficient way of particle identification and analysis. Carriers of hip replacement implants are exposed to particulate wear debris close to and even far away from the implantation sites. This could ease the study of particle transport in the human organism because detectable amounts of particulate matter are likely to arrive in distant organs with regard to the implant. A feasible approach for particle analysis down to the level of electron microscopy is presented here.
\end{abstract}

Loosening of hip replacement implants, but even normal use causes abrasion setting free particles from the interacting surfaces (Fig. $1 \mathrm{a}$; [1]). Wear debris is accumulating near by and in inguinal lymph nodes (ILN). Moreover, implant wear debris in liver, spleen and paraaortal lymph nodes was found [2] suggesting systemic distribution. To study their transport and consequences of particle distribution, these structures were focused on in different organs. Since this is like seeking after a pin in a haystack, a protocol for their efficient localisation and analysis had to be elaborated.

It was found that light microscopy (LM) for the visualisation of most of the wear debris (Fig. 1 b), excluding polyethylene particles, had to be completed by transmission electron microscopy (TEM; EM 912 Omega from LEO Elektronenmikroskopie $\mathrm{GmbH}$, Oberkochen, Germany) for two reasons: i) particles in the range of 10-100 nanometer $(\mathrm{nm})$ can form aggregates which would mimic single particles in the LM (e.g.: aggregation of approx. $1 \mu \mathrm{m}$ in Fig. $1 \mathrm{c}$ ), as well as small single particles (e.g.: $66 \mathrm{~nm}$ particle in c) cannot be resolved by LM; ii) elemental analyses can better be performed on the EM level. Therefore, targets as identified by LM have to be further processed for TEM by embedding and cementing the LM section of interest to the surface of an EM specimen and sectioning it for EM use. TEM and additional elemental analyses are feasible now because EM types like the EM 912 Omega are energy-filtering TEMs (EFTEMs) with this extra-option of elemental analysis. This is necessary for the proof of tracer elements within particles being suspicious of originating from the implant. Since normal EM grids are symmetric, defining the particles' positions was the objective. The so-called finder grid (Fig. $1 \mathrm{~d}$ ) allows to relocate a structure to be analysed later on. Finally, elemental analysis based on energy-filtering TEM (EFTEM) can be performed with the same LEO EM 912 Omega. 


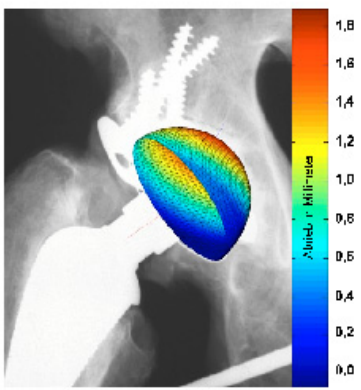

(a)

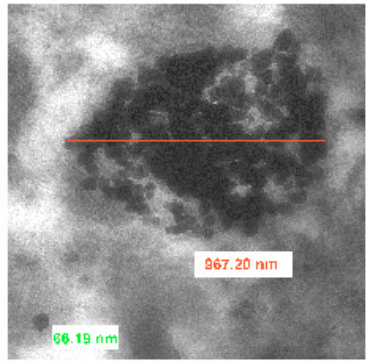

(c)

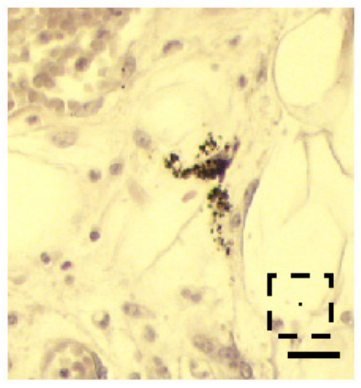

(b)

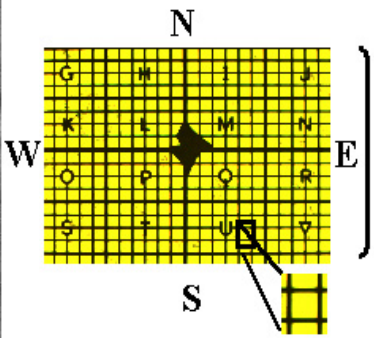

(d)

Fig. 1. Search for and characterisation of prosthesis wear particles in soft tissues. (a) Wear particles' origin: projection of an implant's acetabulum (ac) onto an x-ray image from a total hip prosthesis. Abrasion varies from high (ac, proximal) to low intensity (ac, distal; values, right, in $\mathrm{mm}$ ), is found on both the head and the ac, and where the implant was cemented into the bone. (b) Target structures: LM specimen (ILN) from the body of an implant carrier showing an aggregation of dark particulate debris (centre). Regarding dimensions, the dashed square (right bottom) corresponds to one grid mesh area (emphasized in d) and the black dot in its centre covers the image area in c; bar: $55 \mu \mathrm{m}$. (c) Hard particulate matter: EM section of an ILN from a hip implant carrier (sector width: $1.4 \mu \mathrm{m}$ ). The particle aggregation (centre) would be resolved as one particle by LM; the single particle in the left bottom would be invisible. (d) Recovery of structures for TEM: LM image of a finder EM grid (Maxtaform, Plano, Wetzlar, Germany); the right parenthesis symbol spans $1 \mathrm{~mm}$ (grid diameter: $3 \mathrm{~mm}$ ). Letters define interesting positions (symbolized by compass points $\mathrm{N}, \mathrm{W}, \mathrm{S}, \mathrm{E}$ ); extension piece (right bottom) emphasizes one mesh (width: $60 \mu \mathrm{m}$ ).

\section{References}

1. Schwarz M.L.R., Jörder F., Pustornakova K., Menges S., Milz S., Knaak W., Ritter A., Kaiser E., Moriggl B., Selinger T., Claus A., Scharf H.-P.: Determination of femoral head penetration and main wear direction of autopsy retrieved well-functioning total hip replacements using a $3 \mathrm{~d}$-coordinate measuring machine. $50^{\text {th }}$ Annual Meeting of the Orthopaedic Research society. March 7-10, 2004, San Francisco, USA, Poster presentation.

2. Urban, R.M., Jacobs, J.J., Tomlinson, M.J., Gavrilovic, J., Black, J., Peoc'h, M.: Dissemination of wear particles to the liver, spleen, and abdominal lymph nodes of patients with hip or knee replacement. J. Bone Joint Surg. Am. 82 (2000) 457-476

We thank Prof. V. Mersch-Sundermann (Univ. Giessen, Germany) and Prof. W. Probst (Essingen, Germany) for support and helpful discussions. 